\title{
Vioxx: lessons for Health Canada and the FDA
}

$\mathrm{L}$ ast fall the COX-2 inhibitor rofecoxib (Vioxx), a heavily marketed and successful drug with a low risk of gastrointestinal bleeding, was withdrawn from the market because of an increased risk of cardiovascular disease, mainly myocardial infarction and stroke. ${ }^{1}$ The drug was originally approved by the FDA (and Health Canada) in 1999, despite evidence in the original clinical trials of a nonstatistically significant increase in risk of cardiovascular events and despite the known potential for cardiovascular events associated with any drug that interferes with cyclooxygenase- 2 enzyme regulators of prostacyclin - a potent vasodilator and inhibitor of platelet aggregation.

Why did it take 4 years for the increased risk of serious cardiovascular adverse events to emerge? It has now become clear that both the FDA and (by inference) Health Canada were aware of the increased risk of cardiovascular adverse events long before the drug was withdrawn from the market. There is also email evidence that the manufacturer tried to play down the risk in promotional material for doctors. In the wake of these revelations, Merck's market value dropped US\$28 billion almost overnight, and a sideshow of lawsuits began.

There was no need to fast-track approval of this me-too COX-2 inhibitor (celecoxib had already been approved). Moreover, the regulators did not take into account the fact that the risks, if real, would likely be magnified once the drug came into general use.

Both the FDA and Health Canada put their emphasis and resources into assessing drug benefits, not harms. The bar for approval is low, requiring only that the agent be more effective than placebo. Pre-marketing approval trials are too small to flush out all of the risks of a drug. The built-in bias toward approving drugs without adequate assurance of their safety and with only a fragmentary and underfunded mechanism for postapproval surveillance based on physician reporting of isolated adverse events is a fundamental and (often literally) fatal flaw. Even a simple cumulative meta-analysis $\stackrel{0}{2}$ of postapproval ongoing trials of rofecoxib would have revealed - by December 2000, 4 years before the drug was withdrawn - a statistically significant excess risk of cardiovascular events associated with rofecoxib. ${ }^{2}$

Physicians and patients are aware that no drug is riskfree. Physicians are increasingly aware of the interplay of relative and absolute risks, especially when drugs move out of the controlled confines of the RCT into the Wild West of clinical practice. ${ }^{3}$ Relative benefit and risk rates shown in trials may change, but absolute benefits and risks rates will change: patients in practice are older and sicker than those in clinical trials. Physicians and patients need accurate measures of adverse event frequency.

Using an active surveillance system that targeted serious adverse events would have sounded the alarm much earlier. Both the FDA and Health Canada have failed miserably in carrying out this important aspect of their public mandates. Their current emphasis on partnerships with industry and rapid drug approval conflicts with the public's expectation that these agencies exist to protect them by restricting approval to drugs that have been thoroughly tested and are likely to be free of serious risks. The shift in balance from benefit to harm that can occur when a drug is in widespread use means that regulatory agencies should also shift their priorities. But can they?

The costs and difficulties of postmarketing surveillance are not trivial, and large numbers of patients are needed to allow the detection of rare (but important) adverse events. Nonetheless, we need to find sensible ways to proceed, such as Laupacis and colleagues' recent proposal to obtain postmarketing surveillance data using provincial databases and pharmacare programs. ${ }^{4}$

The FDA and Health Canada have demonstrated their structural inability to do ongoing safety monitoring of new drugs and devices, and industry is far too conflicted to be able to carry out this important task. We need new national agencies to monitor drug safety independently from the approvals process. Only then can physicians and patients be assured an unbiased safety assessment of the drugs they are prescribing and taking. - CMAf

\section{References}

1. Sibbald B. Rofecoxib (Vioxx) voluntarily withdrawn from market. CMAf 2004;171(9):1027-8.

2. Jüni P, Nartey L, Reichenbach S, Sterchi R, Dieppe PA, Egger M. Risk of cardiovascular events and rofecoxib: cumulative meta-analysis. Lancet [serial online]. November 5, 2004 Available: http://image.thelancet.com/extras /04art10237web.pdf (accessed 2004 Nov 26).

3. Barratt A, Wyer PC, Hatala R, McGinn T, Dans AL, Keitz S, et al. EvidenceBased Medicine Teaching Tips Working Group. Tips for learners of evidence-based medicine: 1 . Relative risk reduction, absolute risk reduction and number needed to treat. CMAf 2004;171(4):353-8.

4. Laupacis A, Paterson JM, Mamdani M, Rostom A, Anderson GM. Gaps in the evaluation and monitoring of new pharmaceuticals: proposal for a different approach. CMA7 2003;169(11):1167-70. 